\title{
In-vivo assessment of a case of cutaneous sarcoidosis using reflectance confocal microscopy*
}

\author{
Paola Pasquali ${ }^{1}$, Salvador Gonzalez ${ }^{2}$, Angeles Fortuño ${ }^{3}$, Azael Freites-Martinez ${ }^{4}$
}

DOI: http:/ / dx.doi.org/10.1590/abd1806-4841.20197315

\begin{abstract}
Reflectance confocal microscopy (RCM) is a noninvasive imaging technique that allows visualization of the epidermis and papillary dermis with cellular-level resolution. Granulomatous reactions such as sarcoidosis could be assessed using RCM. The identification of bright beaded-like structures that could correspond to reticulin fibers overlying granulomas, in association with dermoscopy, may be a very useful approach in the diagnosis of sarcoidosis and for the differentiation of this granulomatous entity with superficial cutaneous metastasis.
\end{abstract}

Keywords: Histology, Comparative; Microscopy, Confocal; Sarcoidosis

A woman in her seventies presented for examination and referred multiple firm asymptomatic papules and nodules on her back, which suddenly appeared during the last 2 months. The lesions were asymptomatic, with slow growth, and no significant change, bleeding, or itch. There was no history of fever, weight loss or any other systemic symptoms. Polarized dermoscopy (using DermLite; 3Gen LLC, Dana Point, California, USA) of these papules and nodules showed light-white scales on the surface and sparse, dotted vessels, overlying a homogenous pinkish background (Figure 1). RCM (using Vivascope 1500, Lucid-Tech, Inc., Henrietta, NY, USA; MAVIG GmbH, Munich, Germany) showed in the papillary dermis few bright beaded-like structures with increase of vascularization and prominent inflammatory infiltrate (Figure 2). A tuberculin skin test was negative. Blood examination and serum angiotensin-con-

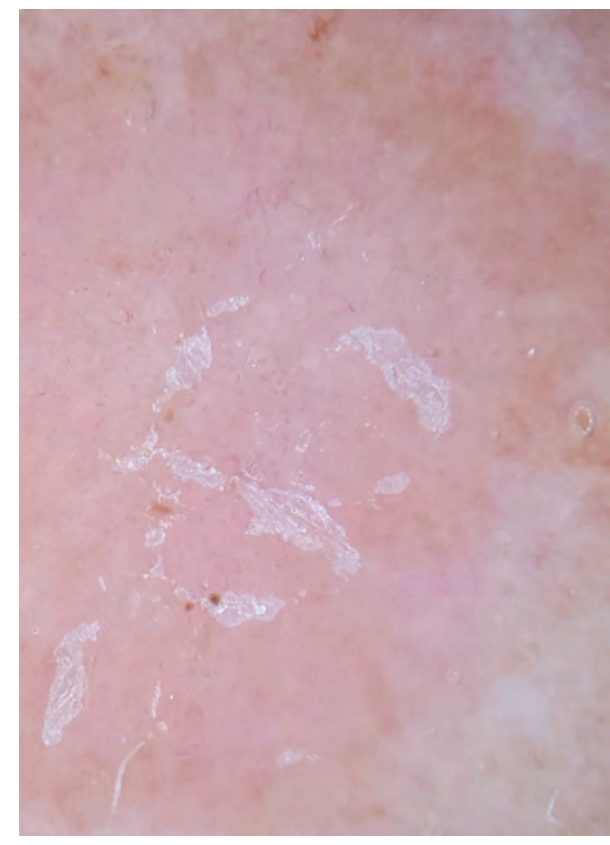

FIGURE 1:

Dermoscopy of cutaneous sarcoidosis. Light-white scales in the surface and sparse, dotted vessels, overlying a homogenous pinkish background

Received 22 May 2017

Accepted 21 January 2018.

* Work conducted at the Dermatology Department, Pius Hospital de Valls, Tarragona, Spain.

Financial support: None.

Conflict of interest: None.

Dermatology Department, Pius Hospital de Valls, Tarragona, Spain.

Dermatology Service, Department of Medicine, Memorial Sloan Kettering Cancer Center, New York, United States Of America.

Eldine Pathology Laboratory, Tarragona, Spain.

Dermatology Service, Department of Medicine, Memorial Sloan Kettering Cancer Center, New York, United States of America.

MAILING AdDREsS:

Azael Freites-Martinez

E-mail: azaelfreites@yahoo.com

C2019 by Anais Brasileiros de Dermatologia 
verting enzyme level were normal and no reactive markers of inflammation were detected. Chest radiography and contrast-enhanced computed tomography showed no hilar lymphadenopathy. A skin punch biopsy from one of the nodular lesions on her back was performed for fungal, bacterial, and mycobacterial tissue culture, all of them negative for any microorganism, as well as the PAS, Ziehl-Neelsen and Gram stains. Immunohistochemistry was negative for Mycobacterium tuberculosis, leishmania and treponema. Histologic examination revealed epithelioid granulomas with multiple Langhans cells up to reticular dermis, with only a sparse infiltrate
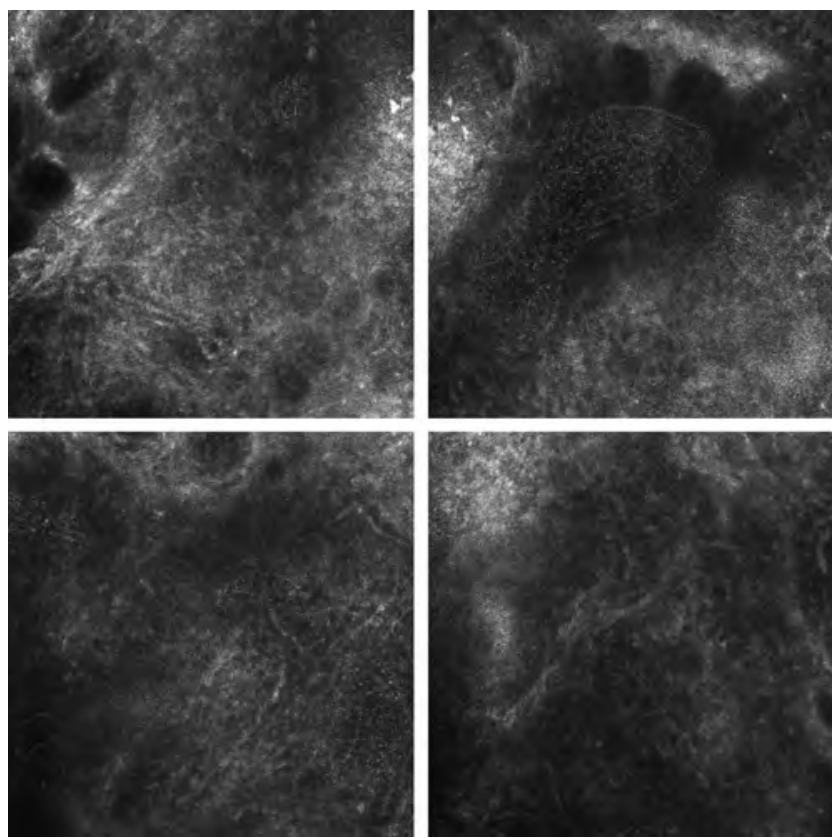

Figure 2: RCM of cutaneous sarcoidosis. Papillary dermis showed bright beaded-like structures overlying granulomas with increase of vascularization and prominent inflammatory infiltrate

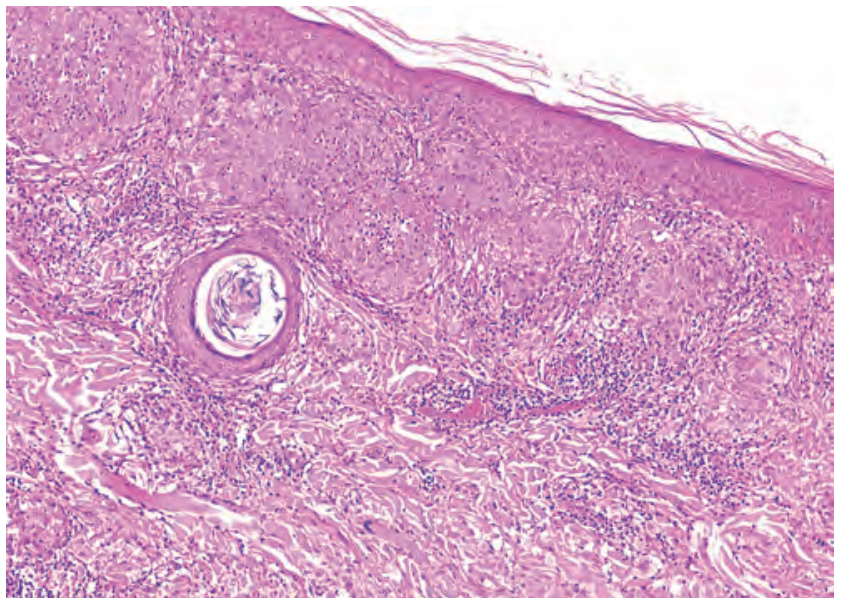

FIGURE 3: Histology revealed epithelioid granulomas with multiple Langhans cells up to reticular dermis, consistent with sarcoidosis (Hematoxylin \& eosin, x200) of lymphocytes at the periphery (Figure 3), reticulin stain showed multiple reticulin fibers surrounding granulomas (Figure 4). The patient was diagnosed with cutaneous sarcoidosis, and treated with clobetasol cream every other day with resolution of the lesions after 2 months of therapy, with residual mild hyperpigmentation and no new lesions with close follow-up.

\section{DISCUSSION}

Sarcoidosis is a non-infectious multisystem granulomatous disorder of unknown etiology characterized by the accumulation of $\mathrm{T}$ lymphocytes, mononuclear phagocytes, and noncaseating granulomas in involved tissues. ${ }^{1}$ Cutaneous lesions may present with a variety of morphologies, including papules, nodules, plaques, and infiltrated scars. The diagnosis is made by exclusion and is supported by the recognition of compatible clinical features, the detection of classic histopathologic findings, and the exclusion of other granulomatous disorders. ${ }^{2}$ When present, cutaneous lesions can be a valuable asset in the confirmation of the diagnosis of sarcoidosis. ${ }^{1}$

Dermoscopically, translucent yellow to orange globular-like or structureless areas associated with linear vessels should raise the suspicion of a granulomatous skin disease such as sarcoidosis and cutaneous leishmaniasis. ${ }^{3}$ However, because dermoscopy seems insufficient to accurately differentiate granulomatous entities, established methods such as histopathology remain the state-of-the-art for sarcoidosis diagnosis. ${ }^{2}$

A novel RCM technique including immunostaining in skin biopsies detected a decreased number of small fiber nerves innervating eccrine glands in decreased perspiration areas compared to normal perspiration areas in a patient with systemic sarcoidosis. ${ }^{4} \mathrm{To}$ date, RCM features of nodular lesions are limited in the literature and no precise correlation exists between dermoscopy and histopathology for sarcoidosis diagnosis. However, RCM can be considered a valuable tool for nodules because, in many instances, a thinned epidermis allowed a good visualization of 'informative' diagnostic

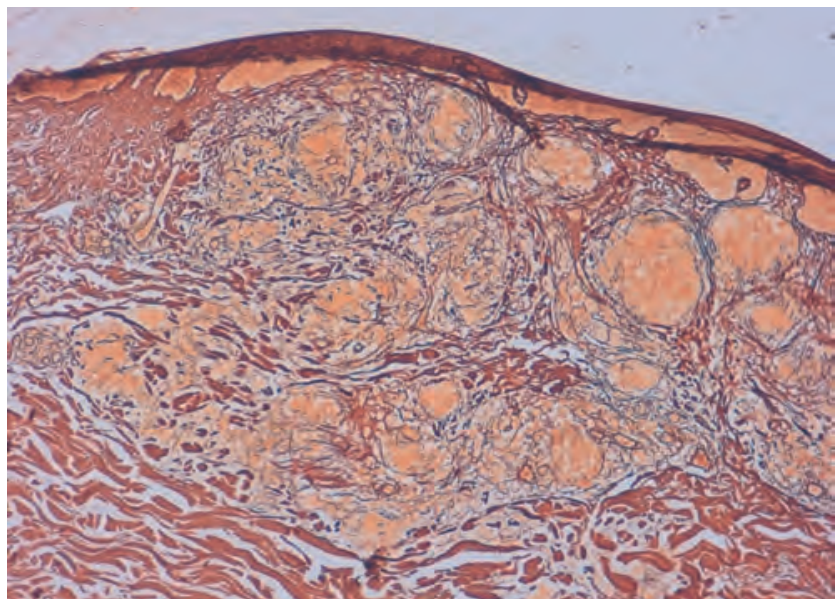

FIGURE 4: Reticulin stain showed multiple reticulin fibers surrounding granulomas (Reticulin stain, x100) 
structures located in the superficial dermis, such as cytological atypia with pleomorphic melanocytes at the dermoepidermal junction level in melanoma metastasis. ${ }^{5}$ Nodular lesions with involvement of the superficial dermis can be meaningfully assessed by RCM. ${ }^{6}$ In this context, granulomatous reactions such as cutaneous sar- coidosis could be assessed using RCM. The identification of bright beaded-like structures with RCM, that could correspond to reticulin fibers overlying granulomas, may be a promising approach in the diagnosis of cutaneous sarcoidosis and for the differentiation of this granulomatous entity with superficial cutaneous metastasis.

\section{REFERENCES}

1. Baughman RP, Lower EE, du Bois RM. Sarcoidosis. Lancet. 2003;361:1111-8.

2. Mangas C, Fernández-Figueras MT, Fité E, Fernández-Chico N, Sàbat M, Ferrándiz C. Clinical spectrum and histological analysis of 32 cases of specific cutaneous sarcoidosis. J Cutan Pathol. 2006;33:772-7.

3. Pellicano R, Tiodorovic-Zivkovic D, Gourhant JY, Catricalà C, Ferrara G, Caldarola $\mathrm{G}$, et al. Dermoscopy of cutaneous sarcoidosis. Dermatology. 2010;221:51-4.
4. Nishida M, Namiki T, Sone Y, Hashimoto T, Tokoro S, Hanafusa T, et al. Acquired anhidrosis associated with systemic sarcoidosis: quantification of nerve fibres around eccrine glands by confocal microscopy. Br J Dermatol. 2018;178:e59-e61.

5. Longo C, Farnetani F, Ciardo S, Cesinaro AM, Moscarella E, Ponti G, et al. Is confocal microscopy a valuable tool in diagnosing nodular lesions? A study of 140 cases. Br J Dermatol. 2013:169:58-67

6. Rajadhyaksha M, González S, Zavislan JM, Anderson RR, Webb RH. In vivo confocal scanning laser microscopy of human skin II: advances in instrumentation and comparison with histology. J Invest Dermatol. 1999;113:293-303.

\section{AUTHORS'CONTRIBUTIONS}

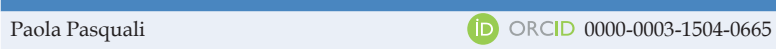

Approval of the final version of the manuscript; Elaboration and writing of the manuscript; Obtaining, analyzing and interpreting the data; Effective participation in research orientation; Intellectual participation in propaedeutic and/or therapeutic conduct of the cases studied; Critical review of the literature; Critical review of the manuscript

Salvador Gonzalez $\quad$ (iD) ORCID 0000-0001-9282-4784

Approval of the final version of the manuscript; Conception and planning of the study; Elaboration and writing of the manuscript; Obtaining, analyzing and interpreting the data; Effective participation in research orientation; Intellectual participation in propaedeutic and/or therapeutic conduct of the cases studied; Critical review of the literature; Critical review of the manuscript

Angeles Fortuño

ORCID 0000-0003-4163-7211

Approval of the final version of the manuscript; Conception and planning of the study; Elaboration and writing of the manuscript; Obtaining, analyzing and interpreting the data; Effective participation in research orientation; Intellectual participation in propaedeutic and/or therapeutic conduct of the cases studied; Critical review of the literature; Critical review of the manuscript

Azael Freites-Martinez

ORCID 0000-0002-3121-5667

Approval of the final version of the manuscript; Conception and planning of the study; Elaboration and writing of the manuscript; Obtaining, analyzing and interpreting the data; Effective participation in research orientation; Intellectual participation in propaedeutic and/or therapeutic conduct of the cases studied; Critical review of the literature; Critical review of the manuscript

How to cite this article: Pasquali P, Gonzalez S, Fortuño A, Freites-Martinez A. In-vivo assessment of a case of cutaneous sarcoidosis using reflectance confocal microscopy. An Bras Dermatol. 2019;94(1):93-5. 